\title{
Plasma interleukin-8 levels are persistently elevated for 1 month after minimally invasive colorectal resection for colorectal cancer
}

\author{
H.M.C. SHANTHA KUMARA ${ }^{1}$, ELLI SUTTON ${ }^{1}$, GEOFFREY A. BELLINI ${ }^{1}$, \\ XIAOHONG YAN ${ }^{1}$, VESNA CEKIC RN ${ }^{1}$, NIPA DILIP GANDHI ${ }^{1}$ and RICHARD L. WHELAN ${ }^{1,2}$ \\ ${ }^{1}$ Division of Colon and Rectal Surgery, Department of Surgery, Mount Sinai West Hospital, New York, NY 10019; \\ ${ }^{2}$ Department of Surgery, Icahn School of Medicine at Mount Sinai, New York, NY 10029, USA
}

Received June 19, 2017; Accepted November 17, 2017

DOI: $10.3892 /$ mco.2017.1538

\begin{abstract}
Minimally invasive colorectal resection (MICR) for colorectal cancer (CRC) is associated with elevated levels of seven proangiogenic proteins that persist for 2-4 weeks after surgery. The proangiogenic plasma may promote tumor growth postoperatively in patients with residual cancer. To the best of our knowledge, the impact of surgery on interleukin 8 (IL-8) levels is unknown. The aim of the present study was to evaluate plasma IL-8 levels after MICR for CRC. Patients with CRC enrolled in an institutional review board-approved plasma/data bank who underwent MICR were eligible. Blood samples were taken preoperatively (preop) and at multiple postoperative (postop) time points, and were stored at $-80^{\circ} \mathrm{C}$. Only patients for whom preop, postop day (POD) 1, POD 3 and at least 1 late postop plasma samples (POD7-34) available were enrolled. Clinical, demographical and pathological data were collected. IL-8 levels were determined via ELISA and results were reported as the mean and \pm standard deviation. The Wilcoxon signed rank test was used for analysis with $\mathrm{P}<0.05$ used as the significance threshold. A total of 73 CRC patients (colon, 62\%; rectal, 38\%) who underwent MICR (laparoscopic-assisted, 60\%; hand-assisted, 40\%) were studied. The mean preop IL-8 level was $20.4 \pm 10.6$ $\mathrm{pg} / \mathrm{ml}$. Significant elevations in plasma IL-8 levels were noted compared with preop levels on POD1 (43.1 \pm 38.6 ; $n=72$; $\mathrm{P}<0.0001)$, POD 3 (33.0 $\pm 30.1 ; \mathrm{n}=71 ; \mathrm{P}<0.0001)$, POD7-13 (29.9 $\pm 21.9 ; \mathrm{n}=50 ; \mathrm{P}<0.0001), \mathrm{POD} 14-20(33.1 \pm 18.3 ; \mathrm{n}=24$; $\mathrm{P}=0.002)$, and for the POD21-27 time point $(24.0 \pm 9.2 ; \mathrm{n}=16$; $\mathrm{P}=0.002)$. In conclusion, plasma IL-8 levels were significantly elevated from baseline for 4 weeks after MICR for CRC. In conjunction with the other proangiogenic MICR-associated
\end{abstract}

Correspondence to: Professor Richard L. Whelan, Division of Colon and Rectal Surgery, Department of Surgery, Mount Sinai West Hospital, Suite 7B, 425 West, 59th Street, New York, NY 10019, USA

E-mail: richard.whelan@mountsinai.org

Key words: minimally invasive colorectal resection, plasma interleukin-8 blood compositional changes, increased IL-8 levels may promote tumor angiogenesis and growth postop.

\section{Introduction}

Surgery is the mainstay of treatment for patients with colorectal cancer (CRC). Where indicated, chemo- and radiotherapy are also utilized either pre- or postoperatively and have improved the outlook of CRC patients. However, despite these treatments $25-30 \%$ of patients undergoing 'curative' resection develop tumor recurrences from unrecognized tumor microfoci and the majority eventually succumb to the cancer $(1,2)$. Notably, there is both clinical and small animal evidence that the surgical trauma associated with resection of the primary tumor may promote the growth of residual tumor deposits in the weeks following surgery, making the postoperative period a crucial period for cancer patients (3-7). The cause(s) of this surgery-associated tumor stimulation is unclear, however, these effects have been attributed to the transient immunosuppression attendant to major surgery as well as the loss of tumor generated inhibitors of angiogenesis following resection of the primary tumor (8-10). There is also human evidence, reported during the last decade, demonstrating that colorectal resection is associated with proangiogenic plasma protein changes that persist for 3-5 weeks after surgery.

Plasma levels of the following proangiogenic proteins have been shown to be persistently increased after minimally invasive colorectal resection (MICR): Vascular endothelial growth factor (VEGF), angiopoeitin-2 (Ang-2), placental growth factor (PIGF), soluble vascular adhesion molecule-1 (sVCAM-1), monocyte chemotactic protein-1 (MCP-1), human chitinase 3-like 1 (Chi3L1), matrix metalloproteinase-3 (MMP-3), and others (11-16). It has also been shown via in vitro endothelial cell (EC) culture studies that plasma from weeks 2 and 3 after MICR significantly stimulates endothelial cells to proliferate, migrate, and invade when compared to EC culture results obtained with the same patients preoperative plasma $(12,17)$; these EC activities are critical to angiogenesis. It is possible, then, that the long duration proangiogenic plasma protein elevations may promote angiogenesis in residual tumor deposits left behind after resection of the primary tumor.

Interleukin-8 (IL-8), a member of the CXC chemokine family, is a powerful chemoattractant for neutrophils, basophils 
and T cells. IL- 8 has also been shown to promote angiogenesis, tumor growth, and metastasis in several murine models (18). IL-8 is notably upregulated in colorectal and other cancer types and has been shown to promote angiogenesis as well as enhance proliferation and survival of tumor cells through autocrine activation (18-20). In addition exogenous IL-8 has been shown to stimulate tumor cell proliferation (21). IL-8 blockade has been demonstrated to inhibit tumor growth (22). IL-8 also plays a role in and is a marker of tumor progression and metastatic disease for a wide range of cancers including gliomas as well as breast and prostate cancers (23-25). Finally, IL-8 is prominent in keratinocytes after IHC staining; this presence suggests that it is a cell migration and chemotactic factor for keratinocytes, suggesting that IL-8 plays a role in wound healing $(26,27)$.

The effect of minimally invasive colon resection (MICR) on postoperative (postop) plasma IL-8 levels is unknown. If IL-8 levels are elevated postop, IL-8 may contribute to the proangiogenic state of plasma following surgery and may also directly promote tumor cell growth in patients with residual tumor post resection. The purpose of this study was to assess plasma levels of IL-8 in CRC patients during the first 5 weeks following MICR.

\section{Patients and methods}

Study population. CRC patients who underwent elective MICR at Mount Sinai West Hospital (formerly Roosevelt Hospital) between October 2007 and April 2012 who had been enrolled in an IRB approved multicenter prospective tissue and data bank were eligible for the present study. The broadly stated purpose of this tissue banking effort was to study the physiologic, immunologic, and oncologic ramifications of major abdominal surgery. Enrolled patients underwent minimally invasive laparoscopic-assisted or hand assisted laparoscopic surgery alone and did not receive a novel drug or other therapy. The indications and type of surgery as well as the demographic, operative, and short-term recovery data were prospectively collected for all the patients. Recently transfused patients, immunosuppressed patients (medication-related and $\mathrm{HIV}^{+}$), and those who received radio- or chemotherapy within 6 weeks of surgery were excluded. Patients undergoing urgent or emergent surgery were, similarly, excluded. Clinical, demographic, and operative data were obtained from this IRB approved data bank [Mount Sinai School of Medicine IRB no.: GCO no. 1:16-2619 (0001)] and the hospital and office charts as well as operative and pathology records.

Blood sampling and processing. Only those CRC MICR patients for whom adequate preoperative (preop) and postoperative (postop) plasma specimens were available were eligible. As per the tissue banking protocol research dedicated blood samples were obtained preoperatively, on postoperative days (POD) 1, 3, and, if possible, at one or more time late point(s) beyond POD 7. Only those patients for whom adequate volumes of plasma were available for the preop, POD 1, POD 3, and 1 late time point (or more) were enrolled. As post discharge blood samples were taken at the time of follow-up office visits, it was not possible to obtain the late specimens on the same postoperative days, thus it was necessary to 'bundle' the late samples into 7 day time blocks (POD7-13, POD14-20, POD21-27, and POD 28-34) that were each considered as single time points. Specimens were collected in heparin-containing tubes, and were processed within 5-6 h of collection. After centrifugation at $450 \mathrm{x} \mathrm{g}$ for $10 \mathrm{~min}$, the plasma was frozen and stored at $-80^{\circ} \mathrm{C}$ until the assays were performed.

IL-8 determination. Plasma IL-8 levels were analyzed in duplicate using a commercially available enzyme-linked immunoabsorbant assay ( $\mathrm{R}$ and D Systems, Minneapolis, MN, USA) according to the manufacturer's instructions. IL-8 concentrations $(\mathrm{pg} / \mathrm{ml})$ were calculated using a standard curve made for every assay and were reported as mean \pm SD for PreOp vs. PostOp IL-8 comparisons.

Statistical analysis. Demographic and clinical data are expressed as the mean \pm SD for continuous variables. In the analysis of preop vs. postop IL-8 levels in CRC patients, the Wilcoxon signed rank test was used for analysis. Comparisons of IL-8 levels of male vs. female patients, the hand-assisted vs. laparoscopic subgroup and patients who had postoperative complications vs. patient who had no post-operative complications were carried out using the Mann Whitney test. Correlation between postop plasma IL- 8 levels and age, incision size and length of surgery was assessed by the Spearman's rank correlation coefficient (rs). Data analysis was performed using SPSS version 15.0 (SPSS, Inc., Chicago, IL, USA).

\section{Results}

A total of $73 \mathrm{CRC}$ patients (36 males, 37 females; mean age $65.8 \pm 12.8$ years) who underwent MICR were included in the study. Of the 73 patients, 45 patients $(62 \%)$ had colon cancer, while 28 patients $(38 \%)$ had rectal malignancies. The majority of patients underwent laparoscopic-assisted resection (60\%), whereas the remainder $(40 \%)$ underwent a hand-assisted or hybrid laparoscopic procedure. The breakdown of operations performed was as follows: Right colectomy, 31.5\%; LAR/anterior resection, 30.1\%; transverse/left colectomy, $16 \%$; sigmoid/rectosigmoid, $12.3 \%$; and other $11 \%$. The mean incision length was $7.75 \pm 3.51 \mathrm{~cm}$ and the mean length of stay was $6.35 \pm 2.6$ days (Table I). The final cancer stage breakdown was as follows: Stage I, 27.5\%; Stage II, 27.5\%; Stage III, $41 \%$; and Stage IV, $4 \%$. Ten different types of complications were noted ( $\mathrm{n}$ for each complication in parentheses): Pleural effusion ( $n=1), C$-Diff colitis $(n=1)$, ileus $(n=8)$, urinary retention $(n=6)$, urinary tract infections $(n=2)$, superficial wound infection $(n=1)$, seroma $(n=1)$, pulmonary hypertension $(n=1)$, tachycardia $(n=1)$, and small bowel obstruction $(n=1)$. Some patients had more than 1 complication. There were no perioperative deaths.

Comparison of preop vs. postop IL-8 plasma levels in CRC patients. The mean preop IL-8 level was $20.4 \pm 10.6 \mathrm{pg} / \mathrm{ml}$ $(n=73)$ (Fig. 1). When compared to preop levels, significant elevations in the mean plasma IL-8 levels $(\mathrm{pg} / \mathrm{ml})$ were identified on POD1 (43.1 $\pm 38.6 ; n=72, \mathrm{P}<0.0001)$, POD 3 (33.0 $\pm 30.1, n=71, P<0.0001), P O D 7-13 \quad(29.9 \pm 21.9, n=50$, $\mathrm{P}<0.0001)$, POD14-20 (33.1 $\pm 18.3, \mathrm{n}=24, \mathrm{P}=0.002)$, and for the POD 21-27 (24.0 $\pm 9.2, \mathrm{n}=16, \mathrm{P}=0.002)$. There was no 
Table I. Demographic and clinical characteristics of the plasma IL-8 study population.

\begin{tabular}{lc}
\hline Characteristic & Value $(\mathrm{n}=73)$ \\
\hline Age, years (mean \pm SD) & $65.77 \pm 12.73$ \\
Sex $(\mathrm{n})$ & \\
Male & $36(49.3 \%)$ \\
Female & $37(50.7 \%)$ \\
Incision length, cm (mean \pm SD) & $7.75 \pm 3.51$ \\
Operative time, min (mean \pm SD) & $302.1 \pm 128.3$ \\
Length of stay, days (mean \pm SD) & $6.35 \pm 2.6$ \\
Type of resection $(\%)$ & \\
Right colectomy & $23(31.5)$ \\
Transverse colectomy & $6(8.2)$ \\
Left colectomy & $5(6.8)$ \\
Sigmoid/rectosigmoid & $9(12.3)$ \\
LAR/AR & $22(30.2)$ \\
APR & $4(5.5)$ \\
Subtotal/total & $4(5.5)$ \\
Surgical method (\%) & \\
Laparoscopic-assisted & $44(60.3)$ \\
Hand-assisted/hybrid laparoscopic & $29(39.7)$ \\
\hline
\end{tabular}

IL-8, interleukin-8; SD, standard deviation; LAR, lower anterior resection; AR, anterior resection; APR, abdominoperineal resection.

significant difference in plasma levels noted on POD 28-34 (vs. preop). As the ' $\mathrm{n}$ ' for the POD 1 and later time points was $<73$ and unique for each time point, the preop baseline level for each of these time points was somewhat different. This is reflected in Fig. 1, which provides in bar graph form the mean preop baseline level for each postop time point. The percentage increase from baseline at each time point was: POD 1, 111\%; POD 3, 63\%; POD 7-13, 53\%; POD 14-20, 45.0\%; POD 21-27, 42\%; and POD 28-34, $24 \%$.

There was no statistically significant correlation found between age and IL- 8 levels preoperatively or at any of the post-operative time points. Similarly there was no significant statistical difference found between male and female patients as regards plasma IL-8 levels preoperatively or at any post-operative time point. Of note, no significant correlation was found between cancer stage and preoperative IL-8 plasma levels. To assess whether incision size (i.e., extent of abdominal wall trauma) correlated directly with the extent of the IL-8 elevation noted postoperatively, the hand-assisted laparoscopic subgroup's $(\mathrm{n}=29$, mean incision length $10.54 \mathrm{~cm} \pm 3.19)$ mean IL-8 levels at each time point were compared to the laparoscopic-assisted subgroups results $(n=44$, mean incision length $5.63 \mathrm{~cm} \pm 2.29$ ). The hand-assisted groups mean IL-8 levels were significantly higher than the laparoscopic-assisted groups results on POD $1(\mathrm{P}=0.02)$ and $3(\mathrm{P}=0.017)$, however, there were no significant differences noted for the POD 7-13, POD 14-20, POD 21-27, and POD 28-34 time points.

As mentioned, there were 23 complications noted in this patient population. Plasma IL-8 levels of patients who

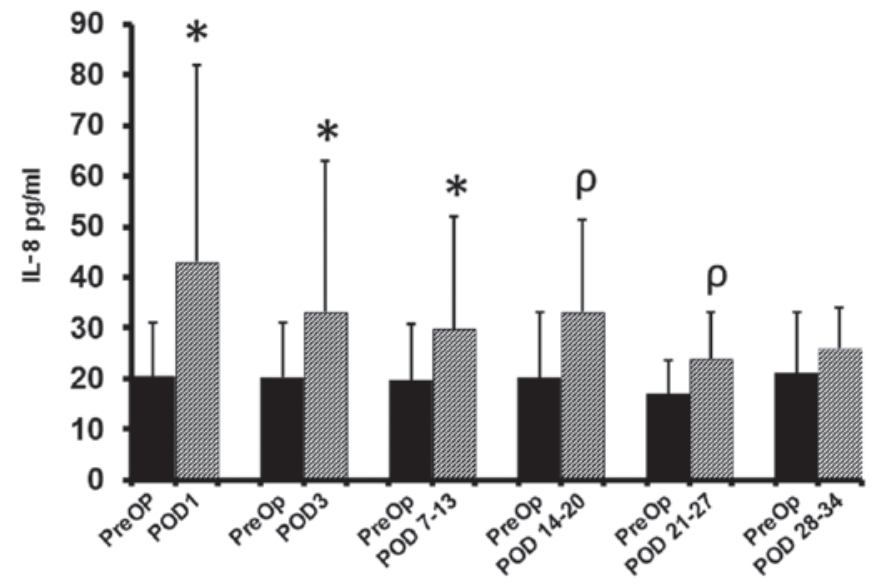

Figure 1. ELISA determined preoperative (PreOp) and postoperative plasma interleukin-8 (IL-8) levels of colorectal cancer patients. IL-8 levels are expressed as mean \pm SD. *PreOp vs. postoperative day (POD) $1(n=72)$, PreOp vs. POD 3 ( $n=71)$, PreOp vs. POD 7-13 (n=50) $\mathrm{P}<0.0001$; ${ }^{\circ}$ PreOp vs. POD 14-20 ( $n=24)$; PreOp vs. POD 21-27 $(n=16) P=0.002$; POD 28-34 time point (ns).

developed complications were compared to the levels identified in the larger subgroup which had no complications. As the ' $\mathrm{n}$ ' for all but 2 of the complications was low, it was not possible to do individual analyses for each complication, therefore, the patients with complications were considered together as a group and compared to the no-complication group. On POD 3 the postoperative IL- 8 level was significantly higher in the sub-group of patients who had complications vs. the no postoperative complication sub-group (30.5 CI 23.8:75.5; $\mathrm{n}=14$ patients vs. $21.8 \mathrm{CI}$ 18.1:27.9; $\mathrm{n}=57, \mathrm{P}=0.039$ ). There were no significant differences identified between the complication group and the no-complication group at the other 4 postop time points, however, the complication groups median IL-8 levels were slightly higher at several of the postop time points.

\section{Discussion}

The vast majority of surgery-related blood protein alterations are short-lived with a duration of $6 \mathrm{~h}$ to 5 days, as is the case for CRP, IL-6, IL-2, EGF, HGF, IGFBP-3 and others (28-30). Most of the short duration blood compositional changes are related to the acute phase inflammatory response to surgical trauma and anesthesia. The present study demonstrates that unlike the above proteins, postop plasma IL-8 levels are significantly elevated from the preop baseline for up to 4th weeks after MICR for CRC. Of note, the percentage change from baseline for the mean plasma IL-8 levels was relatively large, between 45 and $111 \%$, for the first 4 postop time points assessed (through the 3rd week following surgery).

As mentioned earlier, over the last decade it has been found that there is a group of proteins that demonstrate similar patterns of sustained elevation in plasma levels during the first month after surgery. Notably, although these proteins each have numerous actions and effects, all play some role in the complex process of angiogenesis. IL-8, although better known for its other effects, has been shown to promote tumor angiogenesis in murine and in vitro studies $(18,19)$. Additionally, 
Li et al showed that MMP-2 and MMP-9 expression in EC's were enhanced by recombinant human IL-8, suggesting that IL-8 is involved in the degradation of extracellular matrix which facilitates EC migration, invasion, and capillary tube organization (31). Lastly, indirect evidence that IL-8 plays a role in angiogenesis can be found in experimental data demonstrating that IL-8 confers resistance to anti-VEGF therapy as regards colon cancer (32). Thus, it is not altogether surprising that IL-8 joins the group of 7 proteins listed in the introduction that all exhibit sustained increases during the first month after surgery. However, what the etiology of the IL-8 increase after surgery is remains to be determined.

Various types of cancer including colon have been shown to express IL-8 (CXCR8) and its receptors CXCR1 and CXCR2 (23-25). Although IL-8 levels have been shown to be elevated in colorectal cancer patients, the tumor, once removed, cannot account for the postoperative elevation. Since IL-8 plays a role in angiogenesis as well as keratinocyte chemotaxis, it is possible that the healing surgical wounds may be the source of the added protein. Both angiogenesis and the attraction of keratinocytes to a surgical site are critically important to wound healing and, thus, the levels of IL- 8 in the wound may be notably increased. If this is the case then IL- 8 may diffuse from the wounds into the bloodstream. It is also possible that the etiology of the IL-8 increase may vary at different postop time points. The fact that IL-8 levels directly correlated with incision length on POD 1 and 3 (hand vs. lap. assisted comparison) but not for the rest of the 1st month after surgery is consistent with there being a different early mechanism. A likely candidate is the short-lived acute inflammatory response that occurs after major surgery and has been shown to directly correlate with incisional length $(29,30)$. Perhaps after the acute response the healing wounds are the principal source of the added IL-8 in the blood stream. There is evidence that the healing wounds may be the source of the long duration increase in plasma VEGF levels noted after surgery, mentioned above. Wound levels of VEGF have been found to be much higher than plasma levels in a variety of surgical patients (33-35). The authors are currently conducting several studies investigating plasma and wound levels of the above listed proteins after MICR and hope to settle this issue.

The possible ramifications of persistently elevated IL-8 plasma levels after surgery remain to be elucidated. It is possible that the growth of residual tumor deposits may be stimulated since IL- 8 has been shown to promote tumor growth in numerous experimental studies $(19,20,23)$. It is also possible that IL-8, via different mechanisms, may encourage tumor angiogenesis $(18,19)$. There is EC in vitro cell culture evidence that plasma from the second and third weeks after MICR or open surgery for CRC stimulates EC's to proliferate, migrate, and invade at a significantly higher rate when compared to results from EC cultures into which the same patients preoperative plasma was added $(12,17)$. Those results suggest that the net effect of the sustained elevations of IL-8 and the other proangiogenic proteins may encourage angiogenesis during the early postoperative period. It is the authors' hypothesis that tumor angiogenesis may be stimulated in patients with residual cancer following surgery.
Direct evidence for this hypothesis is provided by Peeters et al study of patients with synchronous CRC and liver metastases that noted that $\mathrm{CRC}$ resection (liver lesions left intact) was associated with an increase in the intra- and peri-tumoral vascular density of pre-existing liver metastases 6-12 weeks after surgery (36). In addition, 2 serial PET scan studies of similar patients with synchronous disease noted that the mean and max SUV values of liver metastases were significantly increased 6-12 weeks after CRC resection; of note, in a control group of patients that received no treatment, SUV values were not appreciably changed $(37,10)$. Since FDG uptake, reflects, to some extent, tumor vascularity, these studies indirectly support the above hypothesis. Additional clinical evidence linking the persistent elevation of IL-8 and the other proangiogenic proteins to enhanced tumor growth after MICR is needed before it will be reasonable to conclude that these surgery related changes have clinical import. If surgery is truly associated with a period of enhanced tumor growth then it behooves us to develop anti-cancer treatments that can be given safely to cancer patients in the perioperative period. Such agents must inhibit tumor growth but not interfere with wound healing.

Weaknesses of the present study include the relatively small number of late post-operative plasma samples and the need to bundle the late samples into 7 day time blocks for the statistical comparisons. The logistical impossibility of scheduling outpatient follow-up visits on a particular postoperative day as well as the fact that most patients returned to the office only once during the first 3 weeks after surgery are the reasons for the lower 'n's for the late time points and the need to bundle the specimens. This study concerned only patients who underwent minimally invasive surgery (either laparoscopic-assisted or hand-assisted laparoscopic). No patients in this study group had open CRC surgery. At our center approximately $85 \%$ of colorectal resections are performed using MIS methods. Further research is planned to evaluate perioperative plasma IL- 8 levels of CRC patients who undergo open colorectal resection once blood samples for an adequate number of open resection patients are accumulated in the tissue bank. The final cancer stage for the vast majority (97\%) of the patients included in this study was Stage 1, 2, and 3. In all of these cases the resections were judged to be R-0 resections, based on pathologic assessment of the specimens. In the three Stage 4 patients, 2 had liver metastasis and 1 had abdominal wall metastasis. In these Stage 4 patients the primary tumor was resected and the resections were judged to be R-0 in all cases. Of note, in the 3 Stage 4 patients, the residual tumor may express IL- 8 postoperatively and contribute to the postop plasma elevations. When the 3 Stage 4 patients are excluded, the overall results are not altered. Further research is required to investigate plasma IL-8 levels in patients with R-1 resection. Another weakness is that there is insufficient intermediate and late oncologic follow-up data for the study population to permit an assessment of whether the extent of postop IL-8 elevation correlates with recurrence rates or overall survival.

In summary, the present study has demonstrated that MICR for CRC is associated with significant elevations in the plasma concentrations of IL-8 when compared to preoperative baseline levels. Increased blood levels persisted for the first 
month. The etiology of these changes is uncertain; however, the acute inflammatory response and the healing wounds may play a role. The clinical ramifications of the IL-8 changes are unclear. The IL-8 findings when considered in light of the prior demonstration that plasma levels of at least 7 other proangiogenic proteins are similarly increased raises the possibility that tumor growth may be stimulated during the first month after surgery in patients with residual but unknown metastases. Further investigations to confirm the results of the present study are required.

\section{Acknowledgements}

The present study was made possible by a generous donation from the Wade Thompson Foundation to the Division of Colon and Rectal Surgery, Department of Surgery, Mount Sinai West Hospital, New York, NY, USA.

\section{References}

1. Jagoditsch M, Lisborg PH, Jatzko GR, Wette V, Kropfitsch G, Denk H, Klimpfinger M and Stettner HM: Long-term prognosis for colon cancer related to consistent radical surgery: Multivariate analysis of clinical, surgical, and pathologic variables. World J Surg 24: 1264-1270, 2000

2. Bird NC, Mangnall D and Majeed AW: Biology of colorectal liver metastases: A review. J Surg Oncol 94: 68-80, 2006.

3. Fisher B and Fisher ER: Experimental evidence in support of the dormant tumor cell. Science 130: 918-919, 1959.

4. Peeters CF, de Waal RM, Wobbes T, Westphal JR and Ruers TJ: Outgrowth of human liver metastases after resection of the primary colorectal tumor: A shift in the balance between apoptosis and proliferation. Int J Cancer 119: 1249-1253, 2006.

5. Eggermont AM, Steller EP, Marquet RL, Jeekel J and Sugarbaker PH: Local regional promotion of tumor growth after abdominal surgery is dominant over immunotherapy with interleukin-2 and lymphokine activated killer cells. Cancer Detect Prev 12: 421-429, 1988.

6. Lee JW, Shahzad MM, Lin YG, Armaiz-Pena G, Mangala LS, Han HD, Kim HS, Nam EJ, Jennings NB, Halder J, et al: Surgical stress promotes tumor growth in ovarian carcinoma. Clin Cancer Res 15: 2695-2702, 2009.

7. Coffey JC, Wang JH, Smith MJ, Bouchier-Hayes D, Cotter TG and Redmond HP: Excisional surgery for cancer cure: Therapy at a cost. Lancet Oncol 4: 760-768, 2003.

8. O'Reilly MS, Holmgren L, Shing Y, Chen C, Rosenthal RA, Moses M, Lane WS, Cao Y, Sage EH and Folkman J: Angiostatin: A novel angiogenesis inhibitor that mediates the suppression of metastases by a Lewis lung carcinoma. Cell 79: 315-328, 1994

9. Allendorf JD, Bessler M, Horvath KD, Marvin MR, Laird DA and Whelan RL: Increased tumor establishment and growth after open vs. laparoscopic surgery in mice may be related to differences in postoperative T-cell function. Surg Endosc 13: 233-235, 1999

10. Peeters CF, de Geus LF, Westphal JR, de Waal RM, Ruiter DJ, Wobbes T, Oyen WJ and Ruers TJ: Decrease in circulating anti-angiogenic factors (angiostatin and endostatin) after surgical removal of primary colorectal carcinoma coincides with increased metabolic activity of liver metastases. Surgery 137 246-249, 2005

11. Belizon A, Balik E, Horst P, Feingold D, Arnell T, Azarani T, Cekic V, Skitt R, Kumara S and Whelan RL: Persistent elevation of plasma vascular endothelial growth factor levels during the first month after minimally invasive colorectal resection. Surg Endosc 22: 287-297, 2008.

12. Kumara HM, Feingold D, Kalady M, Dujovny N, Senagore A Hyman N, Cekic V and Whelan RL: Colorectal resection is associated with persistent proangiogenic plasma protein changes: Postoperative plasma stimulates in vitro endothelial cell growth, migration, and invasion. Ann Surg 249: 973-977, 2009 .
13. Shantha Kumara HM, Cabot JC, Yan X, Herath SA, Luchtefeld M, Kalady MF, Feingold DL, Baxter R and Whelan RL: Minimally invasive colon resection is associated with a persistent increase in plasma PlGF levels following cancer resection. Surg Endosc 25: 2153-2158, 2011

14. Shantha Kumara HM, Tohme ST, Herath SA, Yan X, Senagore AJ, Nasar A, Kalady MF, Baxter R and Whelan RL: Plasma soluble vascular adhesion molecule-1 levels are persistently elevated during the first month after colorectal cancer resection. Surg Endosc 26: 1759-1764, 2012.

15. Shantha Kumara HM, Myers EA, Herath SA, Jang JH, Njoh L, Yan X, Kirchoff D, Cekic V, Luchtefeld M and Whelan RL: Plasma monocyte chemotactic protein-1 remains elevated after minimally invasive colorectal cancer resection. World J Gastrointest Oncol 6: 413-419, 2014.

16. Shantha Kumara HM, Gaita D, Miyagaki H, Yan X, Hearth SA, Njoh L, Cekic V and Whelan RL: Plasma chitinase 3-like 1 is persistently elevated during first month after minimally invasive colorectal cancer resection. World J Gastrointest Oncol 8: 607-614, 2016.

17. Shantha Kumara HM, Kirchoff D, Naffouje S, Grieco M, Herath SA, Dujovny N, Kalady MF, Hyman N, Njoh L and Whelan RL: Plasma from the second and third weeks after open colorectal resection for cancer stimulates in vitro endothelial cell growth, migration, and invasion. Surg Endosc 26: 790-795, 2012.

18. Li A, Varney ML, Valasek J, Godfrey M, Dave BJ and Singh RK: Autocrine role of interleukin-8 in induction of endothelial cell proliferation, survival, migration and MMP-2 production and angiogenesis. Angiogenesis 8: 63-71, 2005.

19. Srivastava SK, Bhardwaj A, Arora S, Tyagi N, Singh AP, Carter JE, Scammell JG, Fodstad $\varnothing$ and Singh S: Interleukin-8 is a key mediator of FKBP51-induced melanoma growth, angiogenesis and metastasis. Br J Cancer 112: 1772-1781, 2015.

20. Lee YS, Choi I, Ning Y, Kim NY, Khatchadourian V, Yang D, Chung HK, Choi D, LaBonte MJ, Ladner RD, et al: Interleukin-8 and its receptor CXCR2 in the tumour microenvironment promote colon cancer growth, progression and metastasis. Br J Cancer 106: 1833-1841, 2012.

21. Brew R, Erikson JS, West DC, Flanagan BF and Christmas SE: Interleukin-8 as a growth factor for human colorectal carcinoma cells in vitro. Biochem Soc Trans 25: 264S, 1997.

22. Mian BM, Dinney CP, Bermejo CE, Sweeney P, Tellez C, Yang XD, Gudas JM, McConkey DJ and Bar-Eli M: Fully human anti-interleukin 8 antibody inhibits tumor growth in orthotopic bladder cancer xenografts via down-regulation of matrix metalloproteases and nuclear factor-kappaB. Clin Cancer Res 9: 3167-3175, 2003

23. Yao C, Lin Y, Chua MS, Ye CS, Bi J, Li W, Zhu YF and Wang SM: Interleukin-8 modulates growth and invasiveness of estrogen receptor-negative breast cancer cells. Int J Cancer 121: 1949-1957, 2007.

24. Brat DJ, Bellail AC and Van Meir EG: The role of interleukin-8 and its receptors in gliomagenesis and tumoral angiogenesis. Neuro-Oncol 7: 122-133, 2005.

25. Araki S, Omori Y, Lyn D, Singh RK, Meinbach DM, Sandman Y, Lokeshwar VB and Lokeshwar BL: Interleukin-8 is a molecular determinant of androgen independence and progression in prostate cancer. Cancer Res 67: 6854-6862, 2007.

26. Jiang WG, Sanders AJ, Ruge F and Harding KG: Influence of interleukin-8 (IL-8) and IL-8 receptors on the migration of human keratinocytes, the role of PLC- $\gamma$ and potential clinical implications. Exp Ther Med 3: 231-236, 2012.

27. Sticherling M, Bornscheuer E, Schröder JM and Christophers E: Localization of neutrophil-activating peptide-1/interleukin-8-immunoreactivity in normal and psoriatic skin. J Invest Dermatol 96: 26-30, 1991.

28. Shantha Kumara HM, Tohme ST, Kim IY, Kim DG, Kalady MF, Luchtefeld M, Hoffman K, Dimaggio V and Whelan RL: Minimally invasive colorectal resection is associated with a transient increase in plasma hepatocyte growth factor levels early after surgery for colon cancer. Surg Innov 18: 254-258, 2011.

29. Harmon GD, Senagore AJ, Kilbride MJ and Warzynski MJ: Interleukin-6 response to laparoscopic and open colectomy. Dis Colon Rectum 37: 754-759, 1994.

30. Ordemann J, Jacobi CA, Schwenk W, Stösslein R and Müller JM: Cellular and humoral inflammatory response after laparoscopic and conventional colorectal resections. Surg Endosc 15: 600-608, 2001. 
31. Li A, Dubey S, Varney ML, Dave BJ and Singh RK: IL-8 directly enhanced endothelial cell survival, proliferation, and matrix metalloproteinases production and regulated angiogenesis. J Immunol 170: 3369-3376, 2003.

32. Mizukami Y, Jo WS, Duerr EM, Gala M, Li J, Zhang X, Zimmer MA, Iliopoulos O, Zukerberg LR, Kohgo Y, et al: Induction of interleukin- 8 preserves the angiogenic response in HIF-1alpha-deficient colon cancer cells. Nat Med 11: 992-997, 2005.

33. Karayiannakis AJ, Zbar A, Polychronidis A and Simopoulos C: Serum and drainage fluid vascular endothelial growth factor levels in early surgical wounds. Eur Surg Res 35: 492-496, 2003.

34. Wu FP, Hoekman K, Meijer S and Cuesta MA: VEGF and endostatin levels in wound fluid and plasma after breast surgery. Angiogenesis 6: 255-257, 2003.
35. Wu FP, Hoekman K, Sietses C, von Blomberg BM, Meijer S, Bonjer HJ and Cuesta MA: Systemic and peritoneal angiogenic response after laparoscopic or conventional colon resection in cancer patients: A prospective, randomized trial. Dis Colon Rectum 47: 1670-1674, 2004.

36. Peeters CF, Westphal JR, de Waal RM, Ruiter DJ, Wobbes T and Ruers TJ: Vascular density in colorectal liver metastases increases after removal of the primary tumor in human cancer patients. Int J Cancer 112: 554-559, 2004.

37. Scheer MG, Stollman TH, Vogel WV, Boerman OC, Oyen WJ and Ruers TJ: Increased metabolic activity of indolent liver metastases after resection of a primary colorectal tumor. J Nucl Med 49: 887-891, 2008. 